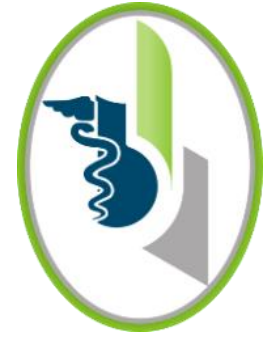

ACCESO ABIERTO

Para citaciones: Espitia, F. (2021). Efecto del Triticum Vulgare intravaginal, en el tratamiento del síndrome genitourinario de la menopausia, en mujeres del Quindío, 2017-2019. Revista Ciencias Biomédicas, 10(3), 152-162.

https://doi.org/10.32997/rcb-2021-3497

Recibido: 3 de marzo de 2021

Aprobado: 2 de mayo de 2021

Autor de correspondencia:

Franklin José Espitia De La Hoz

espitiafranklin@hotmail.com

Editor: Inés Benedetti. Universidad de Cartagena-Colombia.

Copyright: (C) 2021. Espitia, F. Este es un artículo de acceso abierto, distribuido bajo los términos de la licencia https://creativecommons.org/licenses/by-nc$\mathrm{sa} / 4.0 /$ la cual permite el uso sin restricciones, distribución y reproducción en cualquier medio, siempre y cuando el original, el autor y la fuente sean acreditados.

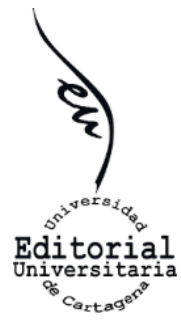

\section{Efecto del Triticum Vulgare intravaginal, en el tratamiento del síndrome genitourinario de la menopausia, en mujeres del Quindío, 2017-2019}

\author{
Effect of intravaginal Triticum Vulgare, in the treatment of \\ genitourinary syndrome of menopause, in women from Quindio, \\ 2017-2019
}

\author{
Franklin José Espitia De La Hoz ${ }^{1}$ (D) \\ ${ }^{1}$ Dirección científica Hathor Clínica Sexológica, Armenia, Colombia.
}

\section{RESUMEN}

Introducción: el principal objetivo terapéutico, en el síndrome genitourinario de la menopausia, es aliviar los síntomas.

Objetivo: determinar el efecto del Triticum vulgare, en el tratamiento del síndrome genitourinario de la menopausia.

Métodos: estudio observacional; incluyó mujeres en posmenopausia, sexualmente activas con diagnóstico de síndrome genitourinario de la menopausia; entre 2017 y 2019, en Armenia (Colombia). El efecto se evaluó mediante la observación de la evolución de la mejoría y desaparición de los síntomas del síndrome genitourinario de la menopausia y con la puntuación del índice de función sexual femenino. Se midió la aparición de reacciones adversas. Se utilizó dosis de $200 \mathrm{mg}$ vía intravaginal diario por cinco semanas, luego cada tercer día por cinco semanas y finalmente una vez a la semana por cinco semanas; se completaron quince semanas de seguimiento.

Resultados: el estudio incluyó 109 mujeres, con edad media de 58,29 \pm 3,17 años. Los síntomas vaginales fueron los más frecuentes, en el $69,72 \%$ de las participantes. Al final del estudio el positivo efecto fue notorio al observarse mejoras significativas en los síntomas, con una disminución de la severidad del 91,74\%, y en la mediana de los síntomas (4 vs. 1, p=0,001). En el índice de función sexual femenino la puntuación final alcanzó 27,16 $\pm 5,29$ con una elevación media de $3,57 \pm 1,18$ puntos $(\mathrm{p}=0,001)$. No se presentaron reacciones adversas.

Conclusión: el Triticum Vulgare intravaginal, mostró un efecto positivo en el tratamiento del síndrome genitourinario de la menopausia. Se requieren ensayos clínicos aleatorizados controlados, sobre su eficacia y seguridad en poblaciones más numerosas.

Palabras Clave: Posmenopausia; Eficacia; Seguridad; Tratamiento Farmacológico; Vaginitis Atrófica. 


\begin{abstract}
Introduction: the main therapeutic objective, in the genitourinary syndrome of menopause, is to alleviate the symptoms.

Objective: to determine the effect of Triticum vulgare in the treatment of genitourinary syndrome of menopause.

Methods: observational study; included sexually active postmenopausal women with a diagnosis of genitourinary syndrome of menopause; between 2017 and 2019, in Armenia (Colombia). The effect was evaluated by observing the evolution of the improvement and disappearance of the symptoms of the genitourinary syndrome of menopause and with the score of the female sexual function index. The appearance of adverse reactions was measured. The $200 \mathrm{mg}$ dose was used intravaginally daily for five weeks, then every third day for five weeks and finally once a week for five weeks; 15 weeks of follow-up were completed.
\end{abstract}

Results: the study included 109 women. The mean age was $58.29 \pm 3.17$ years. Vaginal symptoms were the most frequent, in $69.72 \%$ of the participants. At the end of the study, the positive effect was noticeable as significant improvements were observed in symptoms, with a decrease in severity of $91.74 \%$, and in the median of symptoms ( 4 vs. $1, p=0.001$ ). In the female sexual function index, the final score reached $27.16 \pm 5.29$ with a mean elevation of $3.57 \pm 1.18$ points $(\mathrm{p}=0.001)$. There were no adverse reactions.

Conclusions: intravaginal Triticum Vulgare showed a positive effect in the treatment of genitourinary syndrome of menopause. Randomized controlled clinical trials are required on its efficacy and safety in larger populations.

Keywords: Postmenopause; Efficacy; Safety; drug therapy; Atrophic Vaginitis.

\section{INTRODUCCIÓN}

El síndrome genitourinario de la menopausia (SGUM) agrupa la constelación de signos y síntomas vulvovaginales y urinarios, que se presentan como consecuencia del déficit de estrógenos $(1,2)$. Es una condición crónica, progresiva e irreversible (si no se trata). Lo constituyen los cambios morfológicos y funcionales de los labios mayores y menores, clítoris, vestíbulo/introito, uretra y vejiga; caracterizándolo la sequedad, irritación, molestias sexuales, dolor, disuria, urgencia e infecciones urinarias $(2,3)$.

El SGUM afecta alrededor del 50\% de las mujeres posmenopáusicas, siendo poco probable su mejoría sin tratamiento (1). Sin embargo, las encuestas indican que los profesionales de la salud, rara vez interrogan sobre la sintomatología del SGUM (4), y como resultado, los síntomas no suelen recibir tratamiento, llegando a afectar de forma negativa, la calidad de vida de las mujeres; siendo la sequedad vaginal, el síntoma de mayor prevalencia, y considerándose como el más incómodo por parte de las pacientes $(5,6)$.

El diagnóstico del SGUM se establece con la presencia de al menos dos síntomas o de un signo y un síntoma, los cuales sean considerados como molestos por la paciente, que se encuentren asociados a la menopausia y no secundarios a otra causa $(2,7)$. 
Son varios los estudios y encuestas, que han detallado los efectos negativos del SGUM en la calidad de vida, el bienestar emocional, el funcionamiento y las relaciones sexuales, así como en la imagen corporal de las mujeres postmenopáusicas $(5,8,9)$. En algunos casos, estas mujeres intentan ocultarle los síntomas a su pareja (61\%), sacan excusas para evitar los encuentros sexuales $(42 \%)$ y se sienten menos seguras $(62 \%)$ (10).

El abordaje terapéutico del SGUM consiste en aliviar los síntomas por medio de la terapia hormonal (local y/o sistémica) y/o no hormonal (humectantes y/o lubricantes), así como de cambios en el estilo de vida $(3,11)$. No obstante, la terapia con estrógenos locales vaginales es el "gold standard" en el tratamiento de la sintomatología, ya que ha probado su efectividad para restituir los cambios atróficos vulvovaginales; siendo el tratamiento farmacológico de primera línea recomendado por la NAMS (The North American Menopause Society) $(11,12)$.

El extracto acuoso de Triticum vulgare, aumenta la curación de heridas y ulceras tanto en tejidos cutáneos como no cutáneos, ya que estimula los procesos reparativos tisulares, incita la quimiotaxis y la maduración fibroblástica, además de incrementar el índice fibroblástico, debido a que incentiva la síntesis de ARNm y del ADN en fibroblastos y linfocitos $(13,14)$; lo cual se vierte en una síntesis precoz de tejido de granulación, optimizando los procesos de reepitelización (15).

En la última década se ha incrementado la "cancerofobia", lo que ha llevado al rechazo de la terapia con estrógenos (16); todo por el creciente temor al cáncer de mama $(11,16)$. Dicho rehúso se debe a que tradicionalmente se ha relacionado los estrógenos con la aparición de cáncer (16). De ahí se desprende la necesidad de establecer terapias no hormonales igual de eficaces y más seguras. Es por eso que el objetivo de estudio consistió en determinar el efecto del Triticum vulgare, en el tratamiento del síndrome genitourinario de la menopausia.

\section{MÉTODOS}

Diseño y población. Estudio observacional descriptivo de corte transversal, de mujeres en posmenopausia natural, sexualmente activas en las últimas cuatro semanas con diagnóstico de síndrome genitourinario de la menopausia (SGUM), residentes en el Quindío; entre el 01 de enero de 2017 y el 31 de diciembre de 2019. En una clínica privada de Armenia (Quindío), centro de alta complejidad, de referencia, que atiende población perteneciente al régimen contributivo y subsidiado del Sistema General de Seguridad Social en Colombia. Se excluyeron mujeres con vulvovaginitis de origen infeccioso, antecedentes de cirugía pélvica, enfermedad mental, sangrado vaginal inexplicable, lesión premaligna o maligna vulvovaginal y haber recibido terapia de reemplazo hormonal en los últimos seis meses. Se realizó muestreo por conveniencia.

Procedimiento. Se seleccionaron las mujeres con diagnóstico de SGUM, a partir de la identificación del siguiente código de la clasificación internacional de enfermedades (CIE10): N952-Vaginitis atrófica postmenopáusica. Las mujeres que cumplieron con los criterios de selección y aceptaron participar en el estudio, se les explicaron los objetivos de la investigación, y luego de la firma del consentimiento informado, se diligenció un formulario en Excel 14.0, donde se registraron las características sociodemográficas, los síntomas clínicos y los datos del examen clínico. Se aplicó el Índice de Función Sexual Femenina (IFSF) para la evaluación y seguimiento de la función sexual; el IFSF ha sido traducido al español y validado en diferentes países de habla hispana, entre ellos Colombia.

El instrumento consta de 19 preguntas que evalúan la función sexual femenina en las últimas cuatro semanas. En él se agrupan seis dominios: Deseo (ítems 1-2), Excitación (ítems 3-6), Lubricación (ítems 7-10), Orgasmo (ítems 11-13), Satisfacción (ítems 14-16) y Dolor (ítems 17-19). Cada pregunta tiene 5 o 6 opciones, a la cual se le asigna una puntuación que oscila entre 0 y 5 . El puntaje de cada dominio se multiplica por un factor, $y$ al final el 
resultado es la suma aritmética de los dominios; a mayor puntaje, se espera mejor sexualidad. El rango final de la puntuación del IFSF va desde 2 hasta 36. Una puntuación menor o igual a 26,55, o cuando la puntuación de algún dominio es menor a 3,6 , se considera como criterio de riesgo de disfunción sexual (17-19).

La totalidad de las participantes fueron evaluadas por el investigador principal. El especialista evaluaba la presencia del SGUM, según las molestias referidas por la mujer. El diagnóstico se hizo con el hallazgo de al menos dos síntomas o de un signo y un síntoma descritos en la tabla 1.

A cada paciente se le explicó la naturaleza de su condición clínica, y se le ofreció como alternativa terapéutica el Triticum Vulgare en crema vaginal (extracto acuoso al 20\%). Utilizamos el esquema propuesto en Hathor, Clínica Sexológica, usando la dosis de $200 \mathrm{mg}$ vía intravaginal diario -todas las noches a la hora de acostarse a dormir- durante cinco (5) semanas, seguida de $200 \mathrm{mg}$ intravaginales cada tercer día durante cinco (5) semanas y finalmente $200 \mathrm{mg}$ intravaginales una vez a la semana por cinco semanas, hasta completar un total de 15 semanas de seguimiento. Se realizaron tres (3) seguimientos: a las cinco (5), diez (10) y quince (15) semanas de inicio de la terapia. En cada control se evaluaba la presencia y severidad de la sintomatología y la puntuación del IFSF, además se indagaba la aparición de reacciones adversas.

En el seguimiento se tuvo en consideración, para evaluar el efecto terapéutico, la ausencia o disminución de la sintomatología. Los hallazgos de cada mujer y los resultados del IFSF fueron registrados en cada control, tanto en la historia clínica como en el formulario diseñado por los investigadores. El programa de seguimiento se complementó con una línea telefónica de comunicación, a través de la cual las pacientes podían llamar y comunicar la eventualidad de algún efecto adverso. Los procedimientos de recolección de datos, diligenciamiento y almacenamiento de la información fueron estandarizados mediante capacitación al personal de enfermería que apoyó el estudio, por parte del investigador principal.

A cada una de las mujeres se le preguntó, en cada control, sobre la presencia de la sintomatología del SGUM y se solicitó la calificación de la severidad de cada uno, mediante una escala subjetiva de cuatro grados: "Ninguno" (0), "Leve" (1), "Moderado" (2) y "Grave" (3).

Variables medidas. Sociodemográficas (edad, raza, estado civil, ocupación, nivel de estudios, estrato socio-económico, origen, talla peso, IMC), edad de la menopausia, evolución del tiempo de la menopausia, uso de terapia hormonal de reemplazo, hábitos (ingesta de alcohol, tabaquismo, sedentarismo), antecedentes patológicos, tiempo de convivencia en pareja, variables de comportamiento sexual (masturbación, sexo oral, coito -vaginal o anal-, frecuencia de relaciones sexuales mensuales).

Presencia y severidad de la sintomatología del SGUM y reacciones adversas. Se indagaron además las preguntas de los dominios de la encuesta IFSF.

\section{Análisis estadístico}

Las variables cuantitativas se resumieron con medidas de tendencia central según su distribución, y las variables cualitativas en frecuencias absolutas y proporciones. El análisis se realizó con el programa Epi Info® 7.2. La validación de datos fue realizada en el programa Stata ${ }^{\circledR}$ 15.1. Se utilizó la prueba " $t$ " de Student para comparar variables continuas y $X^{2}$ para variables categóricas.

Aspectos éticos. El estudio fue aprobado por el Comité de Ética de la clínica participante; y cumplió los requerimientos para investigaciones médicas en seres humanos establecidos en la Declaración de Helsinki y con la Resolución 8430 de 1993 por la cual se establecen las normas científicas, técnicas y administrativas para investigación en salud.

Todas las participantes firmaron el consentimiento informado para ingresar al estudio. Se garantizó la confidencialidad de la información. 
Tabla 1. Manifestaciones clínicas del síndrome genitourinario en la menopausia (1-3)

\begin{tabular}{|c|c|c|}
\hline Síntomas vulvovaginales & \multicolumn{2}{|c|}{ Síntomas del tracto urinario inferior } \\
\hline Ardor & \multicolumn{2}{|l|}{ Disuria } \\
\hline Dispareunia & \multicolumn{2}{|l|}{ Nicturia } \\
\hline Dolor & \multicolumn{2}{|l|}{ Polaquiuria } \\
\hline Prurito & \multicolumn{2}{|l|}{ Urgencia urinaria } \\
\hline Disminución de la lubricación vaginal & \multicolumn{2}{|l|}{ Tenesmo vesical } \\
\hline Molestias coitales & \multicolumn{2}{|c|}{ Infecciones urinarias recidivantes } \\
\hline Quemazón & \multicolumn{2}{|l|}{ Incontinencia urinaria } \\
\hline Sangrado postcoital & \multicolumn{2}{|l|}{ Infección postcoital } \\
\hline Sequedad vaginal & \multicolumn{2}{|c|}{ Disminución del flujo de orina uretral } \\
\hline \multicolumn{3}{|c|}{ Signos clínicos } \\
\hline Vulvares & Vaginales & Urinarios \\
\hline Pérdida del vello púbico o escaso y fino. & $\begin{array}{l}\text { Paredes vaginales pálidas, secas y } \\
\text { acartonadas. }\end{array}$ & $\begin{array}{l}\text { Aumento del residuo post- } \\
\text { miccional. }\end{array}$ \\
\hline Pérdida de la almohadilla adiposa labial. & $\begin{array}{l}\text { Alisamiento de los fondos de saco } \\
\text { vaginales. }\end{array}$ & $\begin{array}{l}\text { Disminución de la capacidad } \\
\text { vesical. }\end{array}$ \\
\hline $\begin{array}{l}\text { Retracción y pérdida de la definición de } \\
\text { los labios mayores y menores. }\end{array}$ & $\begin{array}{l}\text { Adelgazamiento de la superficie } \\
\text { vaginal. }\end{array}$ & $\begin{array}{l}\text { Disminución del umbral de } \\
\text { sensibilidad a la distensión de la } \\
\text { vejiga. }\end{array}$ \\
\hline $\begin{array}{l}\text { Acortamiento del prepucio y exposición } \\
\text { excesiva del clítoris. }\end{array}$ & $\begin{array}{l}\text { Petequias, fisuras, ulceración e } \\
\text { inflamación. }\end{array}$ & $\begin{array}{l}\text { Disminución de la presión de cierre } \\
\text { uretral. }\end{array}$ \\
\hline $\begin{array}{l}\text { Disminución de la sensibilidad } \\
\text { Vestibular. }\end{array}$ & Leucorrea y/o secreción anormal. & Meato uretral prominente. \\
\hline Estrechamiento del introito vaginal. & Pérdida de la elasticidad. & $\begin{array}{l}\text { Índice de maduración uretral } \\
\text { anormal. }\end{array}$ \\
\hline $\begin{array}{l}\text { Fusión de los labios y/o pérdida de la } \\
\text { integridad. }\end{array}$ & $\begin{array}{l}\text { Pérdida de los pliegues y rugosidades } \\
\text { vaginales. }\end{array}$ & $\begin{array}{l}\text { Disminución de la presión máxima } \\
\text { de contracción miccional del } \\
\text { músculo detrusor. }\end{array}$ \\
\hline Flujo sanguíneo disminuido. & Disminución de la humedad. & Prolapso uretral, \\
\hline Susceptibilidad a traumas mecánicos. & Adelgazamiento del epitelio vaginal. & \\
\hline Retracción del clítoris. & Aumento sobre 4.6 del $\mathrm{pH}$ vaginal. & \\
\hline \multirow[t]{3}{*}{$\begin{array}{l}\text { Piel hipopigmentada, lisa, no elástica y/o } \\
\text { brillante. }\end{array}$} & $\begin{array}{l}\text { Cambios en la flora facultativa de la } \\
\text { vagina. }\end{array}$ & \\
\hline & $\begin{array}{l}\text { Acortamiento, fibrosis, obliteración } \\
\text { de la cúpula vaginal. }\end{array}$ & \\
\hline & $\begin{array}{l}\text { Cambios en el índice de maduración } \\
\text { vaginal (IMV). }\end{array}$ & \\
\hline
\end{tabular}

\section{RESULTADOS}

De un total de 326 pacientes elegibles, 167 no cumplieron con los criterios de inclusión, por lo que quedaron 159 como población de estudio; de estas, $31(19,49 \%)$ tenían antecedente de cirugía pélvica y $19(11,94 \%)$ presentaron vulvovaginitis de origen infeccioso, por lo que fueron excluidas. Finalmente, el estudio se hizo con 109 mujeres.
La edad media de las participantes fue de 58,29 \pm 3,17 años. El 86,23\% eran de origen urbano, 52,29\% de raza blanca, el $72,47 \%$ estaban casadas o vivían en unión libre, el $61,46 \%$ eran amas de casa y el $77,98 \%$ pertenecían al régimen de aseguramiento contributivo. En la tabla 2 se describen las características sociodemográficas de la población. 
Tabla 2. Características sociodemográficas de las mujeres con SGUM, en el Quindío, 2017-2019

\begin{tabular}{|c|c|}
\hline \multicolumn{2}{|c|}{ Variable y categorías } \\
\hline Edad: $\mathrm{X} \pm \mathrm{DE}$ años & $58,29 \pm 3,17$ \\
\hline Edad de la pareja: $\mathrm{X} \pm \mathrm{DE}$ años & $61,52 \pm 3,86$ \\
\hline Peso: $\mathrm{X} \pm \mathrm{DE} \mathrm{Kg}$ & $64,79 \pm 6,82$ \\
\hline Talla: $\mathrm{X} \pm \mathrm{DE} \mathrm{Cms}$ & $158,35 \pm 4,12$ \\
\hline IMC: $\mathrm{X} \pm \mathrm{DE}$ & $25,73 \pm 6,28$ \\
\hline \multicolumn{2}{|l|}{ Raza } \\
\hline Blancas & $57(52,29 \%)$ \\
\hline Indígenas & $7(6,42 \%)$ \\
\hline Afrocolombianas & $45(41,28 \%)$ \\
\hline \multicolumn{2}{|l|}{ Estado civil } \\
\hline Casadas & $47(43,11 \%)$ \\
\hline Unión libre & $32(29,35 \%)$ \\
\hline Solteras & $17(15,59 \%)$ \\
\hline Viudas & $13(11,92 \%)$ \\
\hline \multicolumn{2}{|l|}{ Ocupación } \\
\hline Amas de casa & $67(61,46 \%)$ \\
\hline Empleadas & $32(29,35 \%)$ \\
\hline Pensionadas & $10(917 \%)$ \\
\hline \multicolumn{2}{|l|}{ Estrato socio-económico } \\
\hline Alto & $33(30,27 \%)$ \\
\hline Medio & $65(59,63 \%)$ \\
\hline Bajo & $11(10,09 \%)$ \\
\hline \multicolumn{2}{|l|}{ Nivel de estudios } \\
\hline Primaria & $8(7,33 \%)$ \\
\hline Secundaria & $34(31,19 \%)$ \\
\hline Técnicos & $39(35,77 \%)$ \\
\hline Profesionales & $28(25,68 \%)$ \\
\hline
\end{tabular}

La edad media de la menopausia fue de 49,51 $\pm 4,82$ años, con una duración media de la menopausia de $9,47 \pm 5,18$ años. El tiempo de convivencia en pareja fue de $14,83 \pm 6,29$ años. La ingesta de alcohol se hizo presente en el 65,13\%. El 17,43\% fumaba, con una mediana de consumo de 3 cigarrillos diarios (rango entre 2 y 7). El sedentarismo se observó en el $71,55 \%$.

En los antecedentes patológicos se encontró que el $38,53 \%$ presentaban HTA, el 33,94\% dislipidemias (56,75\% triglicéridos elevados, y 43,24\% colesterol total elevado, con un 45,94\% de HDL-C bajas), el $18,34 \%$ hipotiroidismo y $8,25 \%$ diabetes tipo 2 . El
$7,33 \%$ tenían antecedente de cáncer de mama, y el $6,42 \%$ presentaban obesidad mórbida.

Respecto a las variables de comportamiento sexual, la masturbación era una experiencia común en el $14,67 \%$ de las participantes. El sexo oral era la práctica sexual preferida por el $87,15 \%$. El coito vaginal lo practicaba el $100 \%$, mientras el anal fue reportado por el 11,92\%. La frecuencia de relaciones sexuales mensuales arrojó una mediana de 2 (rango entre 1 y 4 ).

Los síntomas informados por las pacientes, en orden de frecuencia, se detallan en la tabla 3; siendo la 
sequedad vaginal $(85,32 \%)$ el más frecuente, seguido por la dispareunia $(72,47 \%)$ y la irritación vaginal $(56,88 \%)$. El $74,31 \%$ presentó cuatro o menos síntomas, $19,26 \%$ cinco, y $6,42 \%$ seis o más síntomas; con una mediana de 4 (rango entre 2 y $>7$ ). Los síntomas vaginales fueron los más frecuentes, en el $69,72 \%$ de las participantes; seguido de los sexuales con el $59,63 \%$.

Tabla 3. Sintomatología de las mujeres con SGUM, en el Quindío, 2017-2019

\begin{tabular}{|l|c|}
\hline \multicolumn{1}{|c|}{ Síntomas } & n (\%) \\
\hline Sequedad vaginal & $93(85,32 \%)$ \\
\hline Dispareunia & $79(72,47 \%)$ \\
\hline Irritación vaginal & $62(56,88 \%)$ \\
\hline Prurito (ardor, quemazón) genital & $53(48,62 \%)$ \\
\hline Sinusorragia & $41(37,61 \%)$ \\
\hline Síntomas urinarios & $36(33,02 \%)$ \\
\hline Disminución de la elasticidad de la pared vaginal & $31(28,44 \%)$ \\
\hline Pérdida de los pliegues vaginales & $26(23,85 \%)$ \\
\hline Estrechamiento del introito & $22(20,18 \%)$ \\
\hline Pérdida de las carúnculas himeneales & $19(17,43 \%)$ \\
\hline Tejido frágil & $16(14,67 \%)$ \\
\hline Prolapso uretral & $11(10,09 \%)$ \\
\hline
\end{tabular}

Al inicio del tratamiento, el 75,22\% de las mujeres calificaron la sintomatología como "Grave" disminuyendo a 4,58\% al finalizar el seguimiento, mostrando una mejoría significativa con la terapia del Triticum Vulgare intravaginal $(\mathrm{p}=0,001)$; la "Moderada" pasó del 17,43\% al 3,66\% $(\mathrm{p}=0,001)$, mientras que la "Leve" desapareció a las cinco semanas de tratamiento (tabla 4). Al final del estudio, el positivo efecto se hizo notorio al observarse una disminución en la mediana de los síntomas ( 4 vs. $1 ; \mathrm{p}=0,001)$.

Tabla 4. Seguimiento de la mejoría de la severidad de la sintomatología del SGUM, en mujeres en tratamiento con Triticum Vulgare, en el Quindío, 2017-2019

\begin{tabular}{|l|c|c|c|c|c|}
\hline & & \multicolumn{3}{|c|}{ Triticum Vulgare } & \\
\hline \multicolumn{1}{|c|}{ Severidad } & Basal & 5 semanas & 10 semanas & 15 semanas & p (Basal vs. Final) \\
\hline Ninguna & 0 & 0 & 0 & 0 & 0,001 \\
\hline Leve & $7,33 \%$ & 0 & 0 & 0 & 0,001 \\
\hline Moderada & $17,43 \%$ & $10,09 \%$ & $6,42 \%$ & $3,66 \%$ & 0,001 \\
\hline Grave & $75,22 \%$ & $44,03 \%$ & $16,51 \%$ & $4,58 \%$ & \\
\hline
\end{tabular}

A las cinco semanas de tratamiento, el 55,96\% de las pacientes comunicó mejoría de la sintomatología (tabla 4). El 83,51\%, a las diez semanas de seguimiento, reportó un incremento en la frecuencia coital vaginal, con una mediana de 4 encuentros mensuales (rango entre 2 y 6), disminución de la sequedad vaginal en un $88,07 \%$, de la dispareunia en un $73,71 \%$ y en la irritación vaginal en un $69,72 \%$.

La media en la puntuación total del IFSF fue de $23,24 \pm 1,09$; el puntaje mayor fue de $26,97 \mathrm{y}$ el mínimo de 4,98 puntos; de las cuales el 76,14\% tenían una puntuación menor a 26,55. Al final del 
estudio la puntuación del IFSF alcanzó $27,17 \pm 1,17$ (puntaje mayor de 29,85 y mínimo de 17,46 puntos), con una elevación media de $3,93 \pm 1,13$ puntos $(\mathrm{p}=0,001)$ (tabla 5). La puntuación del IFSF inferior a 26,55 , al final del estudio, se hizo presente en el $11,92 \%$ de las mujeres, observándose una mejoría estadísticamente significativa $(\mathrm{p}=0,001)$.

Tabla 5. Índice de función sexual femenina en mujeres del Quindío con SGUM, en tratamiento con Triticum Vulgare, 2017-2019

\begin{tabular}{|l|c|c|c|c|c|}
\hline & & \multicolumn{3}{|c|}{ Triticum Vulgare } & \\
\hline \multicolumn{1}{|c|}{ Dominios } & Basal & 5 semanas & 10 semanas & 15 semanas & p (Basal vs. Final) \\
\hline Deseo & $3,49 \pm 1,01$ & $3,67 \pm 1,11$ & $4,38 \pm 1,13$ & $4,57 \pm 1,18$ & 0,001 \\
\hline Excitación & $3,52 \pm 1,07$ & $3,61 \pm 1,03$ & $4,37 \pm 1,05$ & $4,65 \pm 1,21$ & 0,001 \\
\hline Lubricación & $3,57 \pm 1,12$ & $3,75 \pm 1,05$ & $4,49 \pm 1,08$ & $4,54 \pm 1,15$ & 0,001 \\
\hline Orgasmo & $3,65 \pm 1,03$ & $3,89 \pm 1,07$ & $4,26 \pm 1,04$ & $4,38 \pm 1,07$ & 0,001 \\
\hline Satisfacción & $4,03 \pm 1,16$ & $4,42 \pm 1,14$ & $4,85 \pm 1,19$ & $4,91 \pm 1,24$ & 0,001 \\
\hline Dolor & $4,98 \pm 1,14$ & $4,74 \pm 1,18$ & $4,43 \pm 1,17$ & $4,12 \pm 1,19$ & 0,001 \\
\hline Total & $23,24 \pm 1,09$ & $24,08 \pm 1,11$ & $26,78 \pm 1,11$ & $27,17 \pm 1,17$ & 0,001 \\
\hline
\end{tabular}

En ninguna de las pacientes se presentaron reacciones adversas, por lo tanto, no se requirió la suspensión de la medicación, y no hubo ninguna pérdida durante el seguimiento (promedio de 14,53 $\pm 1,28$ semanas).

Se identificó falla terapéutica en 5 pacientes (4,58\%); de estas, dos tenían antecedente de cáncer de mama, dos eran fumadoras crónicas y una padecía obesidad mórbida.

\section{DISCUSIÓN}

En la presente investigación, se observó el positivo efecto del uso del Triticum Vulgare intravaginal, para el tratamiento del síndrome genitourinario de la menopausia (SGUM), sin la presencia de efectos adversos.

La terapia estrogénica vaginal local es la recomendación en mujeres que únicamente tienen síntomas vaginales asociados al hipoestrogenismo; ya que pueden revertir los cambios atróficos mientras minimiza la exposición sistémica (20), convirtiéndose en el "gold standard" para el manejo de la sintomatología del SGUM $(12,21)$. Similar a lo observado en este estudio, pero sin los evidentes reacciones adversas de la terapia estrogénica vaginal local.

El uso del láser $\left(\mathrm{CO}_{2}\right.$ fraccionado o Erbio: YAG), se ha constituido en una adecuada terapia no hormonal para el tratamiento del SGUM (22), ya que puede restaurar el epitelio vaginal a un estado similar al estado premenopáusico, aumentar los Lactobacillus, la flora premenopáusica, así como mejorar la puntuación del Índice de Salud Vaginal y los síntomas subjetivos de SGUM, incluidos los síntomas del tracto urinario inferior (23). Aunque nuestro estudio no evaluó los anteriores aspectos, si es concordante al convertirse en otra alternativa no hormonal, al observarse una falla terapéutica de apenas 4,58\%, con una sobresaliente disminución de la severidad de los síntomas "Moderados" y "Graves" al 8,25\% (tabla 4); cuyos positivos resultados, fueron manifiestos.

Nuestros resultados son comparables a los reportados por estudios previos, en los que la eficacia del Triticum vulgare, en patología cervicovaginal diversa, ha sido demostrada (24-26), gracias a su acción reepitelizante; aunque ningunas de esas investigaciones se hizo para el tratamiento del síndrome genitourinario de la menopausia. Por otro lado en una cohorte de 100 mujeres, con edades 
entre 45 y 60 años, a las que se les observó y confirmó por colposcopia y examen citológico, distrofia intensa del epitelio cervicovaginal; Trapani et al. (27), posterior al tratamiento durante 20 días, con un óvulo intravaginal de Triticum vulgare, observaron que el $91 \%$ de las participantes reportaron una remisión completa de los síntomas con mejoría tanto del estado inflamatorio como del trofismo celular, el cual se evidenció mediante los controles colposcópicos y colpocitológicos. Por su parte, De Punzio et al. (25) reportaron un $70 \%$ de remisión completa de la sintomatología, en 30 mujeres posmenopáusicas con síndrome distrófico cervicovaginal, con un $20 \%$ de mejoría del trofismo y $10 \%$ de respuesta poco significativa.

Respecto a la presencia de reacciones adversas, nuestros resultados se encuentran dentro de los porcentajes publicados por Boselli et al. (28), quienes, en un estudio de diseño paralelo abierto, controlado aleatorizado y multicéntrico, de 3 brazos; detectaron $1,1 \%$ de reacciones adversas locales menores, sin la presencia de reacciones adversas graves.

En presencia del conocimiento que se tiene de los potenciales beneficios del Triticum vulgare, donde se sabe que activa los fenómenos de cicatrización y reepitelización, al incitar la mitosis y movilidad de los fibroblastos, con incremento de la capacidad de síntesis de fibras colágenas y glucosaminoglicanos, según lo publicado por Levy et al. (29); es conveniente dirigir la lente hacia investigaciones que incluyan su uso en una condición tan frecuente, en la mujer posmenopáusica, como lo es el síndrome genitourinario de la menopausia (SGUM) (30).

A la luz de los presentes resultados, el papel de los tratamientos no hormonales, en el SGUM, debe evaluarse de forma continua y cuidadosa, en ensayos clínicos controlados aleatorios con mayor número de participantes.

Las fortalezas de este estudio destacan que se trata de la primera investigación, donde el Triticum Vulgare intravaginal es considerado como una opción no hormonal en el tratamiento del SGUM.
Adicionalmente, el importante tamaño de la muestra y el uso de un instrumento estandarizado como lo es el IFSF. En cuanto a las debilidades sobresalen las propias de los estudios observacionales, ya que no se pueden dividir las participantes al azar, pudiéndose crear sesgos o relaciones de causa y efecto donde no existan. Debido a que no se hizo un muestreo aleatorio, las conclusiones aplican solamente para la población estudiada.

En conclusión, el Triticum Vulgare intravaginal, tiene un efecto positivo para el tratamiento de los síntomas del síndrome genitourinario de la menopausia, además de favorecer la puntuación del Índice de Función Sexual Femenino (IFSF); convirtiéndose en una alternativa no hormonal. Su uso se asocia con una importante mejoría, tanto de los síntomas genitourinarios como sexuales. Se requieren ensayos clínicos aleatorizados controlados, para evaluar su eficacia y seguridad, en poblaciones más numerosas, respecto de otras alternativas no hormonales.

\section{AGRADECIMIENTOS}

A las directivas de Hathor, Clínica Sexológica por su apoyo incondicional en este estudio. A la doctora Lilian Orozco Santiago por sus efectivas críticas y acompañamiento en el análisis de los resultados; a las enfermeras de la Clínica La Sagrada Familia por ayudarnos en la búsqueda activa de los datos.

CONTRIBUCIONES DE LOS AUTORES: FE: concepción y diseño del estudio, recolección análisis e interpretación de datos, redacción del borrador del artículo, revisión crítica y aprobación de versión final, responsable de la veracidad e integridad del artículo.

CONFLICTOS DE INTERESES: ninguno que declarar.

\section{REFERENCIAS}

1) The North American Menopause Society (NAMS). The 2020 genitourinary syndrome of menopause position statement of The North American Menopause Society. 
Menopause. 2020; 27(9): $\quad 976-92$ https://doi.org/10.1097/GME.0000000000001609

2) Espitia De la Hoz FJ. Prevalence of genitourinary syndrome of menopause and impact on sexuality of women in Quindío (Colombia), 2013-2016. Rev Colomb Obstet Ginecol 2018; 69(4): 249-59. https://doi.org/10.18597/rcog.3111

3) Gandhi J, Chen A, Dagur G, Suh Y, Smith N, Cali B, Khan SA. Genitourinary syndrome of menopause: an overview of clinical manifestations, pathophysiology, etiology, evaluation, and management. Am J Obstet Gynecol. 2016; 215(6): 704-11. https://doi.org/10.1016/j.ajog.2016.07.045

4) Krychman M, Graham S, Bernick B, Mirkin S, Kingsberg SA. The Women's EMPOWER Survey: Women's Knowledge and Awareness of Treatment Options for Vulvar and Vaginal Atrophy Remains Inadequate. J Sex Med. 2017; 14(3): 425-433. https://doi.org/10.1016/j.jsxm.2017.01.011

5) Moral E, Delgado JL, Carmona F, Caballero B, Guillán C, González PM, Suárez-Almarza J, Velasco-Ortega S, Nieto C; as the writing group of the GENISSE study. Genitourinary syndrome of menopause. Prevalence and quality of life in Spanish postmenopausal women. The GENISSE study. Climacteric. 2018; 21(2): 167-73. https://doi.org/10.1080/13697137.2017.1421921

6) Palma F, Xholli A, Cagnacci A; as the writing group of the AGATA study. The most bothersome symptom of vaginal atrophy: Evidence from the observational AGATA study. Maturitas. 2018; 108: 18-23. https://doi.org/10.1016/j.maturitas.2017.11.007

7) Portman DJ, Gass ML; Vulvovaginal Atrophy Terminology Consensus Conference Panel. Genitourinary syndrome of menopause: new terminology for vulvovaginal atrophy from the International Society for the Study of Women's Sexual Health and the North American Menopause Society. Menopause. 2014; 21(10): 1063-8. https://doi.org/10.1097/GME.0000000000000329

8) Simon JA, Nappi RE, Kingsberg SA, Maamari R, Brown V. Clarifying Vaginal Atrophy's Impact on Sex and Relationships (CLOSER) survey: emotional and physical impact of vaginal discomfort on North American postmenopausal women and their partners. Menopause. 2014; 21(2): 137-42. https://doi.org/10.1097/GME.0b013e318295236f
9) Kingsberg SA, Wysocki S, Magnus L, Krychman ML. Vulvar and vaginal atrophy in postmenopausal women: findings from the REVIVE (REal Women's VIews of Treatment Options for Menopausal Vaginal ChangEs) survey. J Sex Med. 2013; 10(7): 1790-9. https://doi.org/10.1111/jsm.12190

10) Cumming GP, Herald J, Moncur R, Currie H, Lee AJ. Women's attitudes to hormone replacement therapy, alternative therapy and sexual health: a web-based survey. Menopause Int. 2007; 13(2): 79-83. https://doi.org/10.1258/175404507780796424

11) Espitia De La Hoz FJ, Orozco Gallego H. Estriol vs estrógenos conjugados de origen equino en el tratamiento del síndrome genitourinario de la menopausia. Ginecol Obstet Mex 2018; 86(2): 117-26. https://doi.org/10.24245/gom.v86i2.1881

12) North American Menopause Society. The role of local vaginal estrogen for treatment of vaginal atrophy in postmenopausal women: 2007 position statement of The North American Menopause Society. Menopause. 2007; 14(3 Pt 1): 355-69; quiz 370-1. https://doi.org/10.1097/gme.0b013e31805170eb

13) Martini P, Mazzatenta C, Saponati G. Efficacy and tolerability of fitostimoline in two different forms (soaked gauzes and cream) and citrizan gel in the topical treatment of second-degree superficial cutaneous burns. Dermatol Res Pract. 2011; 2011: 978291. https://doi.org/10.1155/2011/978291

14) Romanelli M, Macchia M, Panduri S, Paggi B, Saponati G, Dini V. Clinical evaluation of the efficacy and safety of a medical device in various forms containing Triticum vulgare for the treatment of venous leg ulcers - a randomized pilot study. Drug Des Devel Ther. 2015; 27(9): 2787-92. https://doi.org/10.2147/DDDT.S82712

15) Mollica G, Bonaccorsi G, Martinello R, Minale DG. Evaluation of efficacy and tolerability of fitostimoline vaginal cream (Damor farmaceutici) in the treatment of vaginal inflammation and vulvar dystrophy: A doubleblind randomized controlled trial. Gazz. Med. Ital. Arch. Per. Le Sci. Med. 2008; 167(3): 87-95.

16) Espitia De La Hoz FJ. Efectividad y seguridad del policresuleno en el tratamiento del síndrome genitourinario de la menopausia. Estudio controlado y aleatorizado. Arch Med (Manizales). 2020; 20(2): 28294. https://doi.org/10.30554/archmed.20.2.3743.2020 
17) Rosen R, Brown C, Heiman J, Leiblum S, Meston C, Shabsigh R, Ferguson D, D'Agostino R Jr. The Female Sexual Function Index (FSFI): a multidimensional selfreport instrument for the assessment of female sexual function. J Sex Marital Ther. 2000; 26(2): 191-208. https://doi.org/10.1080/009262300278597

18) Dall'Agno ML, Ferreira CF, Ferreira FV, Pérez-López FR, Wender MCO. Validation of the Six-item Female Sexual Function Index in Middle-Aged Brazilian Women. Rev Bras Ginecol Obstet. 2019; 41(7): 432-439. https://doi.org/10.1055/s-0039-1692694

19) Espitia De La Hoz FJ. Prevalence and characterization of sexual dysfunctions in women, in 12 Colombian cities, 2009-2016. Rev Colomb Obstet Ginecol. 2018; 69(1):921. https://doi.org/10.18597/rcog.3035

20) Kingsberg SA, Krychman ML. Resistance and barriers to local estrogen therapy in women with atrophic vaginitis. J Sex Med. 2013; 10(6): 1567-74. https://doi.org/10.1111/jsm.12120

21) Espitia De la Hoz FJ, Orozco Gallego H. Abordaje diagnóstico y terapéutico del síndrome genitourinario en la menopausia; actualización. Rev Med UCR 2017; 11(2):

67-84.

https://doi.org/10.15517/rmucr.v11i2.34580

22) Arunkalaivanan A, Kaur H, Onuma O. Laser therapy as a treatment modality for genitourinary syndrome of menopause: a critical appraisal of evidence. Int Urogynecol J. 2017; 28(5): 681-5. https://doi.org/10.1007/s00192-017-3282-y

23) Rabley A, O'Shea T, Terry R, Byun S, Louis Moy M. Laser therapy for genitourinary syndrome of menopause. Curr Urol Rep. 2018; 19(10): 83. https://doi.org/10.1007/s11934-018-0831-y
24) D’Antona N, Ricci MG et al. The use of vaginal cream fitostimoline in the therapy of vulvovaginitis and as coadjuvant treatment of diathermocoagulation (DTC) of the portio. Rass Int Clin Ter 1989; 69(19): 873-83.

25) De Punzio C, Bagnoni G, et al. Efficacia del Triticum vulgare nel trattamento post-menopausale della mucosa vaginale. Aggio Sci Ginecol Ostetr 1991; 6:1-4.

26) Santoro MG, Lavazzi B, et al. Trattamento delle vulvovaginiti con fitostimoline: primi risultati. Estratto da V Convergno Nazionale della Societa Italiana di Colposcopia e Patologia Cervico-vaginale, Rimini, 25-27 maggio, 1989.

27) Trapani A, Castobello C, et al. Treatment of cervicovaginal dystrophies with vaginal suppositories of Triticum vulgare and glycolethilen-monophenolic ether. Medicina Oggi 1987; 3(1): 21.

28) Boselli F, Petrella E, Campedelli A, Muzi M, Rullo V, Ascione L, Papa R, Saponati G. Efficacy and tolerability of fitostimoline (vaginal cream, ovules, and vaginal washing) and of benzydamine hydrochloride (tantum rosa vaginal cream and vaginal washing) in the topical treatment of symptoms of bacterial vaginosis. ISRN Obstet Gynecol. 2012; 2012: 183403. https://doi.org/10.5402/2012/183403

29) Levy P, Coriolano A, et al. A clinical evaluation of a new vaginal cream after cervical coagulation. J Bras Ginec 1983; 93(6): 339-44.

30) Espitia-de-la-Hoz FJ. Tratamiento no hormonal del síndrome genitourinario de la menoapusia. Arch Med (Manizales) 2021. 21(2): en prensa. https://doi.org/10.30554/archmed.21.2.3996.2021 